Rapport - Société canadienne d'histoire de l'Église catholique RAPPORT

\title{
L’École ménagère de Roberval, oeuvre de Mère Saint-Raphaël
}

\section{Marie-de-la-Nativité}

Volume 32, 1965

URI : https://id.erudit.org/iderudit/1007336ar

DOI : https://doi.org/10.7202/1007336ar

Aller au sommaire du numéro

Éditeur(s)

Les Éditions Historia Ecclesiæ Catholicæ Canadensis Inc.

ISSN

0318-6148 (imprimé)

1927-7075 (numérique)

Découvrir la revue

Citer cet article

Marie-de-la-Nativité (1965). L’École ménagère de Roberval, oeuvre de Mère

Saint-Raphaël. Rapport - Société canadienne d'histoire de l'Église catholique, 32,

91-100. https://doi.org/10.7202/1007336ar

Tous droits réservés @ Les Éditions Historia Ecclesiæ Catholicæ Canadensis Inc., 1966
Ce document est protégé par la loi sur le droit d'auteur. L'utilisation des services d'Érudit (y compris la reproduction) est assujettie à sa politique d'utilisation que vous pouvez consulter en ligne.

https://apropos.erudit.org/fr/usagers/politique-dutilisation/ 


\title{
L'École ménagère de Roberval, œuvre de Mère Saint-Raphaël
}

\author{
a L'éducation des filles est d'une importance \\ primordiale en tout temps et partout, mais \\ elle prend un caractère plus nécessaire encore, \\ en raison des influences de la femme sur \\ l'orientation et la valeur d'un peuple en \\ formation. D \\ Mgr Victor Tremblay, P.D. \\ Histoire du Saguenay
}

1880 ... C'était encore grande pitié au ROYAUME DU SAGUENAY. La tragédie de 1870 . "le grand feu ", avait balayé le pays de Saint-Félicien à la Baie des $\mathrm{Ha} ! \mathrm{Ha}$ ! et la population était réduite à une extrême pauvreté. Pourtant un vent de patriotisme soufflait sur la Province ... le mot d'ordre était lancé : PRENDRE PIED CHEZ NOUS. $\mathrm{Du}$ haut des chaires et des tribunes, dans la presse et au Parlement, on intensifiait la colonisation; l'élite savante parlait des moyens d'éducation. Au Lac-Saint-Jean, l'Eglise par la paroisse, hâtait la conquête du sol. Mais vers la fin du dix-neuvième siècle, se produit un fléchissement, c'est l'exode rural ${ }^{1}$,

Fort heureusement, M. Elisée Beaudet, député du Saguenay, décide d'enrayer le fléau. Pour lui, comme pour un bon nombre de curés de la région, la colonisation requérait la présence des communautés religieuses : défricher, c'est bâtir, c'est éduquer aussi. On pense à faire venir des Ursulines à Roberval : le projet est négocié avec l'évêque de Chicoutimi, Mgr Dominique Racine, l'Archevêque de Québec, Mgr Taschereau et le chapitre général des Ursulines de Québec.

Chose remarquable, le futur cardinal Taschereau qui s'était opposé à plusieurs demandes de fondations par différents diocèses aux Ursulines du Vieux Cloître de Marie de l'Incarnation, donne sans hésiter son consentement à ce projet qui devra bientôt se réaliser avec le concours de M. le curé Lizotte, de Roberval.

\section{Fondation : 1882}

Il fallait du courage pour accepter à cette époque une fondation à Roberval ... Notre région, c'était une immense forêt coupée de rivières et de lacs; cà et là, quelques villages, des défrichements, des chemins à peine tracés.

1 Alphonse DÉsmets, Histoire de Mère Saint-Raphaël (publié par l'auteur en 1932, Québec). 
Comme Marie de l'Incarnation en 1639, Mère Saint-Raphaël, la fondatrice, accepte d'avance risques et périls, avec la consécration de l'épreuve et la lourde responsabilité d'implanter et de maintenir coûte que coûte, dans un pays nouveau, une œuvre nouvelle. Mais notre Mère était femme à mener très loin les projets les plus périlleux : cette entreprise, elle la dirigera au cours de ses 38 années d'apostolat à Roberval.

Elle s'inspire avant tout de l'esprit de son Ordre et veut agir \& selon les temps et les hesoins » avec prudence et sagesse ${ }^{2}$. Il s'agit de contribuer à l'établissement durable des paroisses, par la formation de la jeune fille, l'épouse et la mère de demain, qui doit réaliser le bonheur du foyer, par l'attachement à l'Eglise et au sol.

De brillantes perspectives étaient réservées à la région : on voyait reculer la forêt, la solitude se peupler, les clochers surgir et grouper par milliers des " faiseurs de terre »... Mais au pays de Maria Chapdelaine, comment résister à la tentation de quitter la dure tâche et d'obéir à la fascination des villes... ? A la femme, incombe le rôle décisif de faire comprendre que la terre est avant tout a la grande Amie ", celle qui ne refuse pas le pain au persévérant labeur.

Mère Saint-Raphaël comprit le rôle social assumé par la fondation du couvent de Roberval et sa pédagogie s'appuiera sur cette nécessité de s'adapter aux besoins des temps présents.

\section{La semence... LES TEMPS HEROIQUES : 1882-1897}

Le 19 mai 1882, les fondatrices disent adieu au cloître de Marie de l'Incarnation et font sur le bateau SAGUENAY le voyage jusqu'à Chicoutimi où elles trouvent le bienveillant accueil des Mères du BonPasteur puis elles prennent en " quatre-roues », la route du Lac-SaintJean. Relais à Hébertville, et le 23 mai, sous une pluie battante, à six heures et demie du soir, arrivée à Roberval. M. le curé Lizotte avait avancé « le Mois de Marie » pour recevoir les Ursulines ${ }^{3}$.

Une maison de bois, inachevée, mal bâtie, de 80 pieds sur 30 , le dos tourné au Lac, voilà le couvent qui les attendait. Pour ameublement, un poêle emprunté, des caisses renversées en guise de chaises, de tables et d'armoires... Comme nourriture, du pain noir et du lard salé. Comme les colons. Il faudra attendre jusqu'en 1888 pour voir entrer la première locomotive à Roberval.

Les Ursulines avaient acheté un terrain au prix de $\$ 400$. Terrain marécageux, non clôturé, bientôt assaini et cultivé, mais il faut protéger la récolte contre les animaux du voisin. Aux amis des premiers jours, on doit les débuts du roulant de la ferme : un quatre-roues à planches, des brebis, don de M. Euloge Ménard, la première vache

2 Sainte-Angèle, onzième legs, cité par Mère Saint-Jean Martin, O.S.U. Esprit de Sainte-Angèle, Rome, 1947, p. 58.

3 Archives des Ursulines de Roberval. 
donnée par l'évêché de Chicoutimi, enfin un cheval, le légendaire « Fan », qui vient de la maison-mère.

Le premier août 1882, eut lieu la bénédiction du couvent. M. l'Abbé Bruno Leclerc, curé l'Hébertville et vicaire forain, avait pris pour texte de son allocution : "La maison que nous élevons est grande "... L'orateur fait voir le nouveau monastère comme le temple de la prière et de l'éducation. En témoignage d'estime et de reconnaissance, la population de la région était accourue : il y eut, ce soir du ler août, feux de joie à Roberval. Déjà une lettre pastorale de Mgr Racine avait fait connaître l'œuvre éducatrice des Ursulines et, comme le dit un de ses biographes, "Mère Saint-Raphaël appartenait à l'Histoire du Lac-Saint-Jean ${ }^{4}$ ». Il n'y avait alors que ce modeste grain de sénevé en bonne terre, mais la Providence veillait : la semence germera et portera fruits...

On lit dans un article de la Revue canadienne de septembre 1882, signé par M. l'Abbé Laflamme : "Les Ursulines ont compris le sens de l'éducation qui leur est confiée... Dans un modeste ouvroir de leur couvent, elles ont installé des rouets, des ourdissoirs et des métiers. Ainsi les élèves de la campagne se familiarisent avec les travaux domestiques. Donner une éducation solide, aussi familiale que possible, telle est la devise de la nouvelle école ". L'observation était juste : le but de la fondatrice était de préparer les élèves à la vie ${ }^{5}$.

Malgré les incommodités multiples, les 75 élèves de 1882 : 25 pensionnaires et 50 externes, reçoivent des leçons pratiques : soins du ménage, tricot, couture, filage et tissage, s'ajoutent au catéchisme, au français, à l'Histoire et au calcul ... Le 7 octobre de la même année, Mère Saint-Raphaël écrit à la Maison-Mère : « Aujourd'hui nous avons commencé à travailler à l'ouvroir. Nos chères enfants sont toutes joyeuses du plan de leur montrer des ouvrages... . La fondatrice ne parle pas encore de l'art culinaire : dans l'unique cuisine de 18 pieds sur 13, qui sert en même temps de boulangerie, un seul poêle suffit à peine pour les pensionnaires et les religieuses ${ }^{6}$.

Une autre lettre datée du 21 décembre fait voir le travail accompli durant les premiers mois : "Nos petites filles du Lac-Saint-Jean ont subi aujourd'hui leur examen devant M. le curé Lizotte... Résultat excellent. Les élèves sont métamorphosées; je me croyais à Québec : même aisance, mêmes manières gracieuses. A l'ouvroir, nos filles ont filé et tissé avec une activité charmante. Avant de partir, M. le curé et M. Euloge Ménard promettent chacun un prix d'Economie domestique.»

A l'exposition de 1883, ce sont les travaux des élèves qui émerveillent les parents : cardes, rouets, dévidoirs et métiers ont chanté leur hymne à la terre, au travail, au foyer de demain.

4 Histoire de Mère Saint-Raphaël.

5 J.C. Laflamme, Les Ursulines au Lac-Saint-Jean, nouvelle de la Revue Canadienne, tome II, no 18, septembre 1882. berval.

o Correspondance de Mère Saint-Raphaël, archives des Ursulines de Ro- 
$\mathrm{La}$ jeune institution fait de rapides progrès. Ecoutons une voix éloquente, celle de M. J. Charles Chapais, agronome, en $1884^{7}$ : "Les Ursulines veulent résoudre le difficile problème de donner aux filles de cultivateurs, la formation ménagère, apanage des femmes de la campagne, afin que l'on cesse de croire que c'est un déshoneur pour un homme instruit de cultiver la terre ... Une ancienne écrira quelques années plus tard : "Elevée dans un couvent de campagne, je n'ambitionnais qu'une chose, " épouser un cultivateur »... Mon mari n'est qu'un guncat, maic j'eanère qu'un jour. il me donnera une ferme. " Et Madame Yvette Olivier-Gouin ajoute : "Quels souvenirs heureux je garde de l'Ecole Ménagère! Nous ne parlions pas d'horticulture, mais il y avait la * semaine au jardin \$... Peu de théorie, en industrie laitière, en art culinaire, en apiculture, mais il y avait a la semaine de la laiterie, le jour du pain, le jour des abeilles, etc. ${ }^{8}$.

Et que dire des belles heures à l'ouvroir ! Certaines élèves apportent leur laine qu'elles filent, tissent en joli droguet pour en confectionner robes ou manteaux. L'une de ces * ferventes $»$, de famille aisée, refuse d'apprendre la musique pour se livrer aux arts ménagers.

Néammoins les programmes et l'horaire de Mère Saint-Raphaël élaborent et maintiennent un sage et constant équilibre entre la formation intellectuelle, artistique, morale, et cette formation ménagère qu'elle veut donner aux jeunes dans un sain épanouissement de leur personnalité féminine.

Bientôt la ruche besogneuse s'avère trop étroite ... On projette la construction de la maison de pierre. Difficultés de transports, manque de ressources, bref, il faudra trois ans avant d'entrer dans le nouveau couvent. En septembre 1890, on recevait 108 pensionnaires. Ere de progrès. La petite ferme avait permis un enseignement agricole. L'honorable Louis Beaubien, ministre de l'Agriculture, accorde en 1892, une subvention pour la construction d'une aile destinée à l'enseignement ménager et pour l'achat d'un lopin de terre à cultiver selon les méthodes modernes de l'époque. Il ajoute $\$ 600$. pour la construction des bâti. ments de la ferme. On multiplie les cours pratiques : une atmosphère d'enthousiasme enveloppe l'école; visites et approbations sont prodiguées à l'œuvre de Mère Saint-Raphaël ${ }^{9}$.

En 1895, à l'exposition de Chicago, on admire des tissus fabriqués à l'Ecole Ménagère de Roberval. Un carton d'exhibits montre le lin à ses différentes étapes : brut, filé, blanchi puis tricoté au crochet en jolis dessins qui défient l'œuvre du temps; des collections de serviettes en fil brut et blanchi, enfin une mœlleuse couverture de laine tissée au métier. * Voilà de la belle ouvrage! » aurait dit Mgr Tessier. Imaginez! l'industrie de la femme canadienne en honneur à Chicago!

7 J.-Chs. Chapais, Réminiscences et revendications, Québec 1910, p. 8, cité par J. C. Tremblay, Mère Saint-Raphaël, Progrès du Saguenay, 1921.

8 Madame Léon Mercier-Gouin (Yvette Olivier), Conférences au Congrès provincial des Sciences ménagères et de l'éducation familiale, Montréal, 1934.

$\theta$ Documents de la Section ménagère, Archives des Ursulines de Roberval. 
En cette même année 1892-93, dans un rapport envoyé par Mère Saint-Raphaël au Surintendant de l'Instruction publique qui en avait fait la demande, nous lisons ce qui suit : * Le cours d'Economie domestique donne large place à la tenue de la maison et à l'art culinaire. Chaque élève apprend à raccommoder ses hardes et doit savoir coudre et tricoter avant d'apprendre les ouvrages de fantaisie. On donne deux leçons hebdomadaires pour la fabrication du beurre et du fromage. Notre ferme couvre 84 arpents dont 66 en culture, d'après une rotation raisonnée en vue de l'industrie laitière ${ }^{10}$.

L'éminent agronome, M. Edouard Barnard, prête une attention constante aux progrès de la ferme. Econome du monastère, Mère SaintRaphaël est encore l'âme dirigeante de cette ferme : plusieurs cultivateurs venant au parloir prendre des leçons d'agriculture, on décerne à notre mère le titre a d'Agronome régionale \$... Le rayonnement de la nouvelle formule d'éducation s'étend plus loin : deux religieuses des Saints Noms de Jésus-Marie séjournent trois semaines à Roberval pour l'étude de nos programmes; de même, Monsieur le Chanoine Beaudet viendra à deux reprises à Roberval avant de fonder, en 1905, son Ecole Ménagère de Saint-Pascal.

En 1895, l'enseignement ménager est installé dans la nouvelle aile et le 15 septembre, c'est la réception à Sir Wilfrid Laurier, premier ministre du Canada. Le journal L'Electeur relate l'événement : - Deux cents élèves étaient en classe et tous les départements d'Economie domestique en activité. Cette Ecole Ménagère est toute une innovation car c'est le seul couvent qui donne un cours complet de ce genre. * Et le journaliste essaie de traduire l'admiration des visiteurs.

Le 28 septembre suivant, c'était l'inauguration officielle sous la présidence de Mgr Laflèche, évêque des Trois-Rivières. Sont présents quatre ministres provinciaux : les honorables Taillon, Beaubien, Chapais, Casgrain; les députés Bellay et Girard ainsi que la plupuart des curés de la région. Cette fois, c'est le Courrier du Canada qui écrit en termes pittoresques : : Le luxe est absent au couvent de Roberval mais on y trouve à profusion de l'air et de la lumière... Heureuse orientation vers l'est avec les brises du Lac. Classes, dortoir, réfectoire, tout respire la gaieté.

Les distingués visiteurs sont ravis par l'Ecole Ménagère et le journaliste précise : * Tout un essaim d'enfants et de jeunes filles se livrent à l'industrie domestique. Une bambine de sept ans tricote toute seule un bas de laine avec une réjouissante fierté. Plus loin, on travaille le lin, le chanvre et la laine. Ici, c'est la beurrerie : cinq ou six jeunes font mouvoir la baratte et le malaxeur : le beurre de première qualité est fabriqué sous nos yeux. "

A la cuisine, nouvel émerveillement : d'adroites mains préparent soupes, viandes et desserts. Le repas sera excellent, tout le monde le

10 Dossier no 2462, Rapport sur le cours d'Economie domestique daté du 9 août 1893, Archives du ministère de l'Agriculture. 
proclame. L'admiration croît devant une superbe exposition des produits de la ferme. Et le Courrier décrit un épisode émouvant : a L'honorable Beaubien tient à la main un diplôme d'honneur et une médaille d'argent. Il annonce que la ferme des Ursulines a obtenu le premier rang dans un récent concours du Mérite agricole." De son long discours, $\mathrm{j}$ 'extrais ce court' passage significatif : "On voit dans l'Histoire, dit-il, que des généraux s'exposent au péril avec leurs soldats pour la gloire de la patrie... Ici se livrent les combats de la civilisation et du progrès: dans cette arène pacifique, les Ursulines sont à l'avantgard̄e. " Le ministre remet ensuite la décoration à Mère Saint-Kaphaël, âme dirigeante de l'exploitation que les Juges du Mérite agricole appellent « ferme modèle ...»

Beaucoup d'autres témoignages seraient à citer ... Ainsi dans la Minerve du 5 janvier 1897, se lit la note suivante : « A L'ECOLE MENAGERE DE ROBERVAL, on accoutume les élèves à régler les dépenses sur les revenus, à organiser des fêtes de familles, bref, à semer le bonheur au foyer ${ }^{11} \ldots$...

\section{Entre les deux feux. Ere de progrès. 1897-1919}

Au lendemain de cet élogieux article de la Minerve, le 6 janvier 1897, le couvent n'était plus qu'un amas de ruines et de cendres. Sous les décombres, l'œuvre de quinze années était anéantie mais c'était peu en regard des sept victimes qui avaient perdu la vie en cette tragédie de quelques heures... Sept religieuses, c'était le quart de la communauté... L'Oeuvre allait-elle survivre ?

Avec l'incendie de 1897, se termine la première phase historique de l'enseignement ménager à Roberval. D'un coup d'œil d'ensemble sur ces quinze années d'héroïsme, nous voyons d'abord une période de défrichement puis c'est la semence féconde. En 1892, l'école sort de l'ombre, jeune et vaillante, puis grandit jusqu'en 1897. De France et de Belgique, on avait demandé les programmes de Roberval. Chaque fois, d'outre-mer étaient venus de hautes approbations. M. le Baron Paul de Vuyst, inspecteur au Ministère de l'Ágriculture, Belgique, félicitait Mère Saint-Raphaël d'avoir compris la nécessité de donner aux futures mères de famille, l'éducation familiale qu'il rêvait pour les femmes belges. N'accusait-on pas la pédagogie de l'époque de paralyser les mains féminines et de centraliser, en le desséchant, le savoir dans les manuels?

Sans doute l'enseignement ménager existait en Europe dans quelques écoles spécialisées mais seuls les programmes de Grenoble et de Lyon étaient venus à la connaissance de Mère S.-Raphaël. Sa correspondance montre qu'elle n'acceptait pas cette spécialisation à \& voie unique »; ce que voulait la fondatrice, c'était une formation aussi complète que possible avec la note agricole en fonction du milieu.

11 "Les Ecoles ménagères 》, nouvelle dans la Minerve, recueillie par Varia Saguenayensia, vol. VIII, p. 85. 
Après l'incendie de 1897, les Annales du monastère disent que nos mères résignées, plus fortes que l'épreuve, sont prêtes à recommencer. L'Ecole Ménagère se doit de donner la leçon de courage et de relèvement dans le malheur... De fait, trois semaines plus tard, en février, les classes reprennent dans une maison d'emprunt. Travail intense, malgré les heures pénibles, qui sera couronné de succès à la fin de l'année scolaire.

Poussée avec activité, la reconstruction permet la rentrée en septembre de la même année. Le rapport annuel mentionne que les travaux sont repris avec entrain à la cuisine, à l'ouvroir et au poulailler. Des bourses sont octroyées à un bon nombre d'élèves et le Gouvernement vote un octroi plus élevé pour l'Ecole Ménagère. Différentes communautés empruntent nos programmes et, grâce aux cours d'été, la formule nouvelle se répand dans la province. Les annales mentionnent que Roberval prend part aux expositions de Paris, en 1900, et de Bruxelles, en 1910.

\section{Evolution - Ecole Ménagère régionale. 1919-1937}

L'événement marquant de l'époque, c'est l'affiliation à l'Université Laval en 1909, ce qui entraîne une nouvelle fusion entre le pensionnat et l'Ecole Ménagère. On adopte les programmes d'étude, section classico-ménagère de l'Université. L'enseignement de la pédagogie au même niveau que les Brevets du Bureau central, permet de préparer les institutrices pour les écoles de la région.

En 1919, Mère Saint-Raphaël précise dans un rapport adressé à l'Honorable Cyrille Delâge, Surintendant de l'Instruction publique, que le cours d'enseignement ménager doit tenir compte du milieu social. Et comme le témoigne encore une ancienne du temps... " Nous apprenions qu'il n'est pas suffisant de donner des bras à la terre mais qu'il faut obtenir par des soins intelligents, une hygiène et une nourriture saines, que ces bras soient assez robustes pour remporter sur la forêt et la plaine des victoires durables."

A cette époque, le nombre d'élèves varie entre 340 et 350 . On vit une ère de prospérité ... mais le Seigneur veut, une fois de plus, apposer le sceau de la croix sur son œuvre. Le 21 janvier 1919, une nouvelle conflagration détruit entièrement l'Ecole Ménagère : les pertes s'élèvent à $\$ 85,000$., alors que les dettes ne sont pas éteintes et que les assurances couvrent à peine le $1 / 5$ des dommages. Amis et protecteurs appuient nos demandes auprès du Parlement... Malgré certains refus, malgré sept ans d'attente, l'œuvre se relèvera, gardant toujours sa vitalité première ${ }^{\mathbf{1 2}}$.

Octogénaire, la vénérée fondatrice est à la dernière année de sa vie... Héroïque couchant d'une vie religieuse dans un beau service

12 Dossier no 1770, Rapport des Ursulines de Roberval, 1920-21, Archives du ministère de l'Agriculture, Québec. 
d'amour. Avec confiance, elle relève les courages. Le 29 décembre 1920, elle s'éteint sans avoir la consolation de léguer à ses filles une œuvre prospère, mais un de ses derniers conseils reste vivant : Q Que chaque religieuse possède les sciences ménagères pour les enseigner et les faire aimer. .

En affirmant que Mère Saint-Raphaël a jeté une semence première, nous croyons rendre hommage à son initiative hardie, comme à son œuvre d'apôtre de la famille, de l'école et de l'Eglise... Oeuvre sociale aussi. Notre mère voulait apporter à l'éducation * la planche de salut * dont parlaient les sociologues et les colonisateurs de ỉ'époque.

Avons-nous conservé l'héritage légué par notre Mère fondatrice? Avons-nous fait fructifier le blé de 1882 en terre saguenéenne? Pour répondre, il nous reste à suivre l'évolution de l'Ecole Ménagère durant ces quarante-cinq dernières années.

Après l'incendie de 1919, il fallait sauvegarder l'œuvre. Avec une dette de $\$ 66,000$., la loi ecclésiastique ne nous permettait pas un emprunt. La population demandait une Ecole Normale à laquelle serait annexé l'enseignement ménager. Il faudra cinq ans avant de réaliser ce projet. En attendant, " on se serre les coudes..." La musique se donne à la communauté, des classes à la chapelle. L'art culinaire persiste dans les réfectoires; la couture et la coupe parfois dans les dortoirs. Les rouets et les métiers descendent du grenier et y remontent; le parloir, c'est la salle à tout faire.

Cet état de gêne n'abat nullement les élans... Encore moins la bonne humeur! On maintient les programmes des Ecoles Ménagères régionales. En 1925, s'ouvrira l'Ecole Normale avec l'étage destiné aux travaux domestiques : on respire à l'aise dans des pièces bien éclairées et aérées... Cuisine, salle de coupe et de tissage AVEC le joli vivoir que n'ont pas oublié les anciennes.

Depuis 1892, l'Enseignement ménager relevait du Ministère de l'Agriculture; en 1929, il est sous le contrôle de l'Instruction publique. La ferme continue son évolution mais n'est plus au service de l'école.

Des activités nouvelles se multiplient... Ainsi cinq ans après l'organisation des Cercles de Jeunes Naturalistes dans la province, le Cercle Saint-Raphaël, en 1936, est affilié à la Société canadienne d'Histoire naturelle, dont le président est le $\mathrm{F}$. Marie-Victorin, E.C. Après 30 ans d'expérience dans l'institution, les C.J.N. sont bien vivants : des groupes d'élèves partent en randonnées et reviennent riches de découvertes. Avec des yeux neufs, on admire les trésors naturels de notre région.

\section{Epanouissement - L'Institut Familial. 1937-1965}

1937 ... Les Ecoles ménagères entrent dans une nouvelle phase de leur histoire sous la dynamique impulsion de Mgr Tessier, visiteur propagandiste. L'école se verra rajeunie : climat épanouissant qui cul- 
tive l'initiative et le sens des responsabilités. On fait large place aux disciplines intellectuelles et morales, en particulier à la pédagogie familiale et à la psychologie de l'enfant. La spiritualité féminine est en honneur : ne prépare-t-on pas des épouses et des mères chrétiennes ?

LES ECOLES DE BONHEUR ont connu l'essor magnifique que vous connaissez et Roberval « est entré dans le vent »... Congrès et réunions, études des programmes nouveaux, ont permis de s'adapter aux directives des temps présents; entre beaucoup d'innovations heureuses, l'organisation des équipes familiales ne reste pas une simple théorie mais devient une belle réalisation dans un climat de ferveur et d'amour.

Avec la construction de 1956, l'Institut familial donne aux édifices scolaires une dimension de 607 pieds... * La maison que nous élevons est grande ... * avait-on dit en 1882. Grande en effet était l'œuvre de Mère Saint-Raphaël, grande encore est cette même ouvre toujours au service de l'éducation.

On n'entend plus la chanson des rouets, mais la formule première est toujours en honneur : donner à la famille, la femme forte dont la société a besoin.

Si Mère Saint-Raphaël revenait, reconnaîtrait-elle son œuvre ? La semence a grandi et porte des fruits nouveaux... Les sciences ménagères sont devenues un secteur, secteur qui doit rester important parmi les autres cours d'étude. Cependant les besoins de 1965 ne sont pas ceux de 1882.

L'Histoire est une grande éducatrice : elle invite à l'audace et au renouvellement; elle fait comprendre les problèmes des âges actuels et elle oblige à repenser nos attitudes en éducation.

Actuellement les Instituts familiaux, tout comme les autres écoles indépendantes du Québec, s'interrogent sur un avenir incertain... Quelles que soient les ORIENTATIONS nouvelles, l'esprit de la Fondatrice doit continuer à inspirer notre respect des personnalités féminines.

Tout comme au siècle dernier, il faut pour l'Eglise et la société des foyers chrétiens. La vie sociale avec ses exigences nouvelles n'est pas la seule responsable des conditions de vie féminine : c'est parce qu'elle doit s'épanouir en maternité que la femme est différente de l'homme. Cette différenciation, Jean XXIII la proclamait, le 6 septembre 1961, lorsqu'il disait : « Méconnaître la diversité des fonctions respectives de l'homme et de la femme finirait par faire déprécier la dignité de la femme.»

Notre trépidant vingtième siècle a-t-il compris cette vérité ? Pourquoi faut-il que tant de femmes s'improvisent épouses et mères alors qu'elles ont préparé durant de longues années un brevet professionnel ? C'est pour pallier ces désastreuses improvisations que furent créées les ECOLES DE BONHEUR ... 
Mère Saint-Raphaël a tracé un premier sillon lumineux : à nous de continuer dans une optique et un contexte qui peuvent être différents, mais qui permettent encore le même idéal, la même foi robuste au service de la plus noble cause...

MÈre Marie-de-LA-Nativité, O.S.U. Professeur de pédagogie et de français, Ecole Normale des Ursulines, Roberval. 